Research Article

\title{
Generalized Jordan N-Derivations of Unital Algebras with Idempotents
}

\author{
Xinfeng Liang $\mathbb{D}$ \\ School of Mathematics and Big Data, Anhui University of Science \& Technology, Huainan 232001, China \\ Correspondence should be addressed to Xinfeng Liang; xfliang@aust.edu.cn
}

Received 29 March 2021; Revised 21 April 2021; Accepted 6 May 2021; Published 9 June 2021

Academic Editor: Ji Gao

Copyright (c) 2021 Xinfeng Liang. This is an open access article distributed under the Creative Commons Attribution License, which permits unrestricted use, distribution, and reproduction in any medium, provided the original work is properly cited.

Let $\mathscr{A}$ be a unital algebra with idempotent $e$ over a 2-torsionfree unital commutative ring $\mathscr{R}$ and $\mathfrak{S}: \mathscr{A} \longrightarrow \mathscr{A}$ be an arbitrary generalized Jordan $n$-derivation associated with a Jordan n-derivation $J$. We show that, under mild conditions, every generalized Jordan n-derivation $\mathfrak{S}: \mathscr{A} \longrightarrow \mathscr{A}$ is of the form $\mathfrak{S}(x)=\lambda x+J(x)$ in the current work. As an application, we give a description of generalized Jordan derivations for the condition $n=2$ on classical examples of unital algebras with idempotents: triangular algebras, matrix algebras, nest algebras, and algebras of all bounded linear operators, which generalize some known results.

\section{Introduction}

Throughout this paper, let $\mathscr{R}$ be a commutative ring with an identity and let $\mathscr{A}$ be a unital algebras over $\mathscr{R}$. Let us assume that $\mathscr{A}$ has an idempotent $e \neq 0,1$ and let $f=1-e$. In this case, $\mathscr{A}$ can be represented in the so called Peirce decomposition form

$$
\mathscr{A}=e \mathscr{A} e+e \mathscr{A} f+f \mathscr{A} e+f \mathscr{A} f,
$$

where $e \mathscr{A} e$ and $f \mathscr{A} f$ are subalgebras with unitary elements $e$ and $f$, respectively, $e \mathscr{A} f$ is an $(e \mathscr{A} e, f \mathscr{A} f)$-bimodule and $f \mathscr{A} e$ is an $(f \mathscr{A} f, e \mathscr{A} e)$-bimodule. It is worth to mention that $\mathscr{A}$ is isomorphic to a generalized matrix algebra [1]. We assume that $\mathscr{A}$ satisfies

$$
\begin{aligned}
\text { exe.eAd } f & =0=f \text { Ae.exe implies exe }=0, \\
\text { eAf } f . f x f & =0=f x f . f \text { Ae implies } f x f=0,
\end{aligned}
$$

for all $x \in \mathscr{A}$. Some special examples of unital algebras with a nontrivial idempotents having the property (\$) are triangular algebras, matrix algebras, and prime (and hence in particular simple) algebras with nontrivial idempotent, nest algebras, standard operator algebras (see [2] for more details). It follows from (\$) that at least one of the bimodules $e \mathscr{A} f$ and $f \mathscr{A} e$ is nonzero.
Let $\mathscr{R}$ be an associate algebra or ring. $\{x, y\}=x y+y x$ is the Jordan product of elements $x, y \in \mathscr{R}$. For any integer $n \geq 1$ and any $x_{1}, x_{2}, \ldots, x_{n} \in \mathscr{R}$. Set $p_{1}\left(x_{1}\right)=x_{1}$ and

$$
\begin{aligned}
p_{n}\left(x_{1}, x_{2}, \ldots, x_{n}\right)= & \left\{p_{n-1}\left(x_{1}, x_{2}, \ldots, x_{n-1}\right), x_{n}\right\} \\
= & p_{n-1}\left(x_{1}, x_{2}, \ldots, x_{n-1}\right) x_{n} \\
& +x_{n} p_{n-1}\left(x_{1}, x_{2}, \ldots, x_{n-1}\right),
\end{aligned}
$$

for $n \geq 2$, which is called the Jordan $n$-product of $x_{1}, x_{2}, \ldots, x_{n}$. Denote by $\varphi: \mathscr{R} \longrightarrow \mathscr{R}$ a linear mapping; we call $\varphi$ a Jordan $n$-derivation if

$$
\varphi\left(p_{n}\left(x_{1}, x_{2}, \ldots, x_{n}\right)\right)=\sum_{k=1}^{n} p_{n}\left(x_{1}, x_{2}, \ldots, \ldots\left(x_{k}\right), \ldots, x_{n}\right),
$$

for all $x_{1}, x_{2}, \ldots, x_{n} \in \mathscr{R}$. It is obvious that Jordan $n$-derivations are usual Jordan derivations for $n=2$; moreover, it is also easily checked that the definition of Jordan 3-derivations are equivalent to the conception of Jordan triple derivations.

A linear mapping $\mathfrak{S}: \mathscr{A} \longrightarrow \mathscr{A}$ is said to be a generalized Jordan $n$-derivation if there exists a Jordan $n$-derivation $J: \mathscr{A} \longrightarrow \mathscr{A}$ such that 


$$
\begin{aligned}
& \mathbb{S}\left(p_{n}\left(x_{1}, x_{2}, \ldots, x_{n}\right)\right) \\
& =p_{n}\left(\mathfrak{S}\left(x_{1}\right), x_{2}, \ldots, x_{n}\right) \\
& \quad+\sum_{k=2}^{n} p_{n}\left(x_{1}, x_{2}, \ldots, J\left(x_{k}\right), \ldots, x_{n}\right),
\end{aligned}
$$

for all $x_{1}, x_{2}, \ldots, x_{n} \in \mathscr{A}$. It is clear that any Jordan 2derivations are usual generalized Jordan derivations.

Note that any Jordan $n$-derivation is an example of a generalized Jordan $n$-derivation. On the other hand, any multiplier function $F(x)=\lambda x$ for all $x \in \mathscr{A}$ and $\lambda \in Z(\mathscr{A})$ is a Jordan example of a generalized Jordan n-derivation.

The motivation for this study comes from the results of [2-6]. Benkovič and Sčirovnik considered the structure of Jordan derivations on unital algebra with nonzero idempotent $e$ and introduced the notation of singular Jordan derivations which comes out to be very important in the study of mappings on unital algebra with nonzero idempotent $e$. It turns out that, under some mild conditions, every Jordan derivation is the sum of a derivation and a singular Jordan derivation. By introducing the concept of Jordan $\mathrm{n}$-derivation ( $\mathrm{n}$ is any positive integer), Qi, Guo and Zhang [5] showed that every multiplicative Jordan $n$-derivation on $\mathscr{A}$ is additive; and furthermore, it turns out that a mapping on $\mathscr{A}$ is a multiplicative Jordan $n$-derivation if and only if it is an additive Jordan derivation. In Ref. [3], Benkovič present a new approach and show that every generalized Lie $n$-derivation $F: \mathscr{A} \longrightarrow \mathscr{A}$ is of the form

$$
F(x)=\lambda x+\Delta(x) \text { for all } x \in \mathscr{A}
$$

where some $\lambda \in Z(\mathscr{A})$ and $\Delta$ is a Lie n-derivation of $\mathscr{A}$. Inspired by the structures of Jordan derivations [3], multiplicative Jordan n-derivations [5], and genelized Lie n-derivations [2] of unital algebras with a nonzero idempotent, the main purpose of this article is to study generalized Jordan $n$-derivation of unital algebras with the property (\$). In the main theorem of the paper, Theorem 1, we show that, under certain mild assumptions, every generalized Jordan $n$-derivation is of the form

$$
\mathfrak{S}(x)=\lambda x+J(x) \text { for all } x \in \mathscr{A},
$$

where $\lambda \in Z(\mathscr{A}), J$ is a Jordan $n$-derivation of $\mathscr{A}$. We shall use some known results about the form of Jordan derivations on unital algebras with nonzero idempotent that were obtained in [2-4]. Let us mention that in different papers [2, 3, 7-11] Jordan derivations on triangular algebras and related algebras were studied from different perspectives.

In this paper, we present a new approach which is analogue to Benkovič's article [3], which elegantly reduces the problem of describing a generalized Jordan $n$-derivation to the describing a Jordan $n$-derivation. It turns out that if S: $\mathscr{A} \longrightarrow \mathscr{A}$ is a generalized Jordan $n$-derivation associated with a Jordan $n$-derivation $J$, then a linear mapping $H=$ S - $J: \mathscr{A} \longrightarrow \mathscr{A}$ satisfies

$$
H\left(p_{n}\left(x_{1}, \ldots, x_{n}\right)\right)=p_{n}\left(H\left(x_{1}\right), \ldots, x_{n}\right),
$$

for all $x_{1}, \ldots, x_{n} \in \mathscr{A}$. Therefore, it suffices to consider linear mappings with property (\$). Under suitable assumptions on unital algebra $\mathscr{A}$ with a nonzero idempotent (Proposition 1 ), any such generalized Jordan $n$-derivation is of the form $H(x)=\lambda x$ for all $x \in \mathscr{A}$ and some $\lambda \in Z(\mathscr{A})$.

\section{Preliminaries and the Main Theorem}

Let $\mathscr{A}$ be a unital algebra with idempotent $e$ and $f=1-e$, which satisfies (\$). For convenience, we shall use the following notations $a=e a e \in e \mathscr{A} e, m=e m f \in e \mathscr{A} f, t=$ fte $\in f \mathscr{A} e$, and $b=f b f \in f \mathscr{A} f$. Thus, every element $x \in \mathscr{A}$ can be represented in the form

$$
x=e a e+e m f+f t e+f b f=a+m+t+b,
$$

where $e \mathscr{A} f$ is an $(e \mathscr{A} e, f \mathscr{A} f)$-bimodule and $f \mathscr{A} e$ is an ( $f \mathscr{A} f, e \mathscr{A} e$ )-bimodule.

Let us list some classical example of unital algebras with idempotent $e$ and $f=1-e$, which satisfies (\$). Since these examples have already been presented in many papers, see $[2,12-14]$, we just state their title without any details.

(a) Matrix algebra $\mathscr{A}=M_{n}(A), n \geq 2$, where $A$ is a unital algebra.

(b) Every simple unital algebra $\mathscr{A}$ with nontrivial idempotent, which satisfies (\$).

(c) Unital prime algebras with nontrivial idempotent.

(d) Triangular algebra $\mathscr{T}=\left[\begin{array}{cc}A & M \\ & B\end{array}\right]$ such that the bimodule $M$ is faithful as a left $A$ and also as a right $B$-module. The most important examples of triangular algebras are upper triangular matrix algebras $T_{n}(A)$ and block upper triangular matrix algebras $B_{n}(A)$ over a unital algebra $\mathrm{A}$ and also nest algebras $T(\mathfrak{N})$, where $\mathfrak{N}$ is a nest in a Hilbert space $\mathbb{H}$.

The first very useful observation refers to the form of the center of $\mathscr{A}$, which is identical to ([15], Proposition 3) and ([16], Lemma 3.1, Lemma 3.2). Throughout the paper, $\mathscr{A}$ denotes a unital algebra with a nonzero idempotents $e$ satisfying (\$).

By [12], [Proposition 2.1], it follows that the center of $\mathscr{A}$ is equal to

$$
\begin{aligned}
& \mathscr{Z}(\mathscr{A})=\{a+b \in e \mathscr{A} e \oplus f \mathscr{A} f a m=m b, n a=b n, \\
& \forall m \in e \mathscr{A} f, \forall n \in f \mathscr{A} e\} .
\end{aligned}
$$

Furthermore, we know that the map $\tau: \mathscr{Z}(\mathscr{A}) e \longrightarrow \mathscr{Z}(\mathscr{A}) f$ is an algebraic isomorphism such that $a m=m \tau(a)$ and $n a=\tau(a) n$ for all $a \in \mathscr{Z}(\mathscr{A}) e, m \in e \mathscr{A} f, n \in f \mathscr{A} e$.

Remark 1. Let $\mathscr{A}$ be a unital algebra with nontrivial idempotent $e$ and $f=1-e$. For any $x \in \mathscr{A}$ and for any integer $n \geq 2$, we have 


$$
\begin{aligned}
p_{n}(x, e, \ldots, e) & =2^{n-1} \text { exe }+\operatorname{exf}+f x e, \\
p_{n}(x, f, \ldots, f) & =2^{n-1} f x f+\operatorname{exf}+f x e .
\end{aligned}
$$

In particular, $\{x, e\}=x e+e x=2 e x e+e x f+f x e$ and $\{x, f\}=x f+f x=2 f x f+e x f+f x e$.

In this section, we will prove the main result, Theorem 1. As we mentioned in the introduction, the problem of a description of a generalized Jordan $n$-derivations can be reduced to a description of a map satisfying (8). Let us begin with the solution of this problem.

Proposition 1. Let $\mathscr{A}$ be a unital algebra with a nontrivial idempotent e satisfying (\$) over a 2-trosionfree commutative ring $\mathscr{R}$. Let us assume that $\mathscr{Z}(e \mathscr{A} e)=\mathscr{Z}(\mathscr{A}) e$ and $\mathscr{Z}(f \mathscr{A} f)=\mathscr{Z}(\mathscr{A}) f$. Let a linear mapping $H: \mathscr{A} \longrightarrow \mathscr{A}$ satisfies

$$
H\left(p_{n}\left(x_{1}, \ldots, x_{n}\right)\right)=p_{n}\left(H\left(x_{1}\right), x_{2}, \ldots, x_{n}\right)
$$

for all $x_{1}, \ldots, x_{n} \in \mathscr{A}$. Then

$$
H(x)=\lambda x \text { for all } x \in \mathscr{A},
$$

where $\lambda \in Z(\mathscr{A})$

Proof. To prove this proposition, we will need the following Claims:

(i) Claim 1. $H(0)=0$. By taking $x_{i}=0$ for all $i=1,2, \ldots, n$, one can show $H(0)=0$.

(ii) Claim 2. With notations as above, we have $e H(e) e \oplus f H(f) f \in Z(\mathscr{A}), \quad H(m)=e H(e) e m=$ $m H(f) f$ and $H(t)=t e H(e) e=f H(f) f t$ for all $m \in e \mathscr{A} f, t \in f \mathscr{A} e$.

By the definition $H$, we have

$$
\begin{aligned}
& 0=H\left(p_{n}(e, f, \ldots, f)\right)=p_{n}(H(e), f, \ldots, f)=2^{n-1} \\
& f H(e) f+e H(e) f+f H(e) e,
\end{aligned}
$$

and

$$
\begin{aligned}
& 0=H\left(p_{n}(f, e, \ldots, e)\right)=p_{n}(H(f), e, \ldots, e)=2^{n-1} \\
& e H(f) e+e H(f) f+f H(f) e,
\end{aligned}
$$

which imply $e H(e) f=f H(e) e=e H(f) f=f H$ $(f) e=0$ and $f H(e) f=e H(f) e=0$ as char $\mathscr{R} \neq 2$. Therefore $H(e)=e H(e) e \in e \mathscr{A} e$ and $H(f)=$ $f H(f) f \in f \mathscr{A} f$.

For all $m \in e \mathscr{A} f$, we have

$$
\begin{aligned}
H\left(2^{n-2} m\right) & =H\left(p_{n}(f, \ldots, f, m)\right)=p_{n}(H(f), \ldots, f, m) \\
& =\left\{p_{n-1}(H(f), f, \ldots, f), m\right\}=2^{n-2} m H(f) f,
\end{aligned}
$$

and

$$
\begin{aligned}
H\left(2^{n-2} m\right) & =H\left(p_{n}(e, \ldots, e, m)\right)=p_{n}(H(e), \ldots, e, m) \\
& =\left\{p_{n-1}(H(e), e, \ldots, e), m\right\}=2^{n-2} e H(e) e m
\end{aligned}
$$

for all $m \in e \mathscr{A} f$. Hence,

$$
H(m)=e H(e) e m=m H(f) f
$$

as char $\mathscr{R} \neq 2$. On the other hand, for all $t \in f \mathscr{A} e$,

$$
\begin{aligned}
H\left(2^{n-2} t\right) & =H\left(p_{n}(f, \ldots, f, t)\right)=p_{n}(H(f), \ldots, f, t) \\
& =\left\{p_{n-1}(H(f), f, \ldots, f), t\right\}=2^{n-2} f H(f) f t,
\end{aligned}
$$

and

$$
\begin{aligned}
H\left(2^{n-2} t\right) & =H\left(p_{n}(e, \ldots, e, t)\right)=p_{n}(H(e), \ldots, e, t) \\
& =\left\{p_{n-1}(H(e), e, \ldots, e), t\right\}=2^{n-2} t e H(e) e .
\end{aligned}
$$

Therefore,

$$
H(t)=t e H(e) e=f H(f) f t
$$

for all $t \in f \mathscr{A}$ e. Combining (18) and (21) with the definition of the center $Z(\mathscr{A})$, we have

$$
e H(e) e \oplus f H(f) f \in Z(\mathscr{A}) .
$$

(iii) Claim 3. With notations as above, we have $H(a)=$ $e H(e) a$ and $H(b)=f H(f) b$ for all $a \in e \mathscr{A} e$ and $b \in f \mathscr{A f}$

For all $a_{11} \in e \mathscr{A l e}$, we have

$$
\begin{aligned}
2^{n-1} H(a)= & H\left(p_{n}(e, \ldots, e, a)\right)=p_{n}(H(e), e, \ldots, e, a) \\
= & \left\{p_{n-1}(H(e), e, \ldots, e), a\right\}=2^{n-2} e H(e) a \\
& +2^{n-2} a H(e) e .
\end{aligned}
$$

Since the characteristic $\mathscr{R}$ is not 2 , this implies

$$
2 H(a)=e H(e) a+a H(e) e=2 e H(e) a,
$$

and then

$$
H(a)=e H(e) a=a e H(e) e
$$

for all $a \in e \mathscr{A} e$.

Similarly, one can check that

$$
H(b)=b H(f) f=b f H(f)
$$

for all $b \in f \mathscr{A} f$.

For all $x=a+m+t+b \in \mathscr{A}$, by Claims $1-3$, we have

$$
\begin{aligned}
H(x)= & H(a)+H(m)+H(t)+H(b)=e H(e) a \\
& +e H(e) m+f H(f) t+f H(f) b=\lambda x,
\end{aligned}
$$

where $\lambda=e H(e) e+f H(f) f \in Z(\mathscr{A})$ 
The main result of the the paper state:

Theorem 1. Let $\mathscr{A}$ be a unital algebra with a nontrivial idempotent e satisfying (\$). Let us assume that

(i) $\mathscr{Z}(e \mathscr{A} e)=\mathscr{Z}(\mathscr{A}) e$

(ii) $\mathscr{Z}(f \mathscr{A} f)=\mathscr{Z}(\mathscr{A}) f$

Then any generalized Jordan $n$-derivation $\mathfrak{S}: \mathscr{A} \longrightarrow \mathscr{A}$ is of the from $\mathfrak{S}(x)=\lambda x+J(x)$ for all $x \in \mathscr{A}$, where $\lambda \in Z(\mathscr{A})$ and $J: \mathscr{A} \longrightarrow \mathscr{A}$ is a Jordan $n$-derivation.

Proof. Let $\mathfrak{S}: \mathscr{A} \longrightarrow \mathscr{A}$ be a generalized Jordan n-derivation associated with a Jordan n-derivation $J$. According to the definition

$$
\begin{gathered}
\mathfrak{S}\left(p_{n}\left(x_{1}, \ldots, x_{n}\right)\right)=p_{n}\left(\mathfrak{S}\left(x_{1}\right), \ldots, x_{n}\right) \\
+\sum_{k=2}^{n} p_{n}\left(x_{1}, \ldots, J\left(x_{k}\right), \ldots, x_{n}\right), J\left(p_{n}\left(x_{1}, \ldots, x_{n}\right)\right) \\
=p_{n}\left(J\left(x_{1}\right), \ldots, x_{n}\right)+\sum_{k=2}^{n} p_{n}\left(x_{1}, \ldots, J\left(x_{k}\right), \ldots, x_{n}\right),
\end{gathered}
$$

for all $x_{1}, \ldots, x_{n} \in \mathscr{A}$. Let us denote $H=\mathfrak{S}-J$. If we subtract upper equalities, we see that a linear mapping $H: \mathscr{A} \longrightarrow \mathscr{A}$ satisfies

$$
H\left(p_{n}\left(x_{1}, \ldots, x_{n}\right)\right)=p_{n}\left(H\left(x_{1}\right), \ldots, x_{n}\right)
$$

for all $x_{1}, \ldots, x_{n} \in \mathscr{A}$. Since all assumption from Proposition 1 are fulfilled there exists $\lambda \in Z(\mathscr{A})$ such that $H(x)=\lambda x+J(x)$.

According to Theorem 1 and ([2], Theorem 4.1), Jordan $n$-derivation is usual Jordan derivation if $n=2$, the following result is immediate.

Corollary 1. Let $\mathscr{A}$ be a unital algebra with a nontrivial idempotent e satisfying (\$). Let us assume that

(i) $\mathscr{Z}(e \mathscr{A} e)=\mathscr{Z}(\mathscr{A}) e$

(ii) $\mathscr{Z}(f \mathscr{A} f)=\mathscr{Z}(\mathscr{A}) f$

Then any generalized Jordan derivation $F: \mathscr{A} \longrightarrow \mathscr{A}$ is of the from $F(x)=\lambda x+J_{1}(x)+J_{2}(x)$ for all $x \in \mathscr{A}$, where $\lambda \in Z(\mathscr{A}), J_{1}: \mathscr{A} \longrightarrow \mathscr{A}$ is a derivation and $J_{2}: \mathscr{A} \longrightarrow \mathscr{A}$ is a singular Jordan derivation.

Corollary 2. ([4], Theorem 3.11) Let $\mathscr{A}$ be a unital algebra with a nontrivial idempotent e satisfying (\$) and a bimodule eAf is faithful as a left eAde-module and as a right $f \mathscr{A} f$-module. Then every Jordan derivation of $A$ can be expressed as the sum of a derivation and an antiderivation.

We apply Theorem 1 to the classical examples of unital algebras: triangular algebras (upper triangular matrix algebras, nest algebras), matrix algebras, and algebras of bounded linear operators. Our main result reduces the description of a generalized Jordan $n$-derivation to the description of a Jordan $n$-derivation.
It is well-known that all Jordan derivations of matrix algebras and prime algebras are derivations [17, 18]. Using the results from ([2], Section 3), one could prove that there are no nonzero singular Jordan derivations of the matrix algebra $M_{n}(A)$ over a unital algebra $A$. One can also obtain that there are no nonzero singular Jordan derivations of a unital prime algebra $\mathscr{A}$ with a nontrivial idempotent $e$. Therefore, Theorem 1 implies the following corollaries.

Corollary 3. Let $\mathscr{A}=M_{s}(A), s \geq 3$, where $A$ is a unital $(s-1)$-torsionfree algebra. Then every generalized Jordan derivation $F: \mathscr{A} \longrightarrow \mathscr{A}$ is of the form $F(x)=\lambda x+J(x)$, where $\lambda \in Z(\mathscr{A}), J: \mathscr{A} \longrightarrow \mathscr{A}$ is a derivation.

We conclude this article with some applications of the main theorem. In case $\mathrm{A}$ is a unital algebra with a nontrivial idempotent $e$ such that $f \mathscr{A} e=0$, and that the bimodule $e \mathscr{A} f$ is faithful as a left e⿻Ae-module and also as a right $f \mathscr{A} f$-module, the algebra $\mathscr{A}$ is a triangular algebra. The triangular algebra $\mathscr{A}$ satisfies (\$) and by the definition $\mathscr{A}$ has no nonzero singular Jordan derivations. Therefore, combining with [7], we obtain the following result.

Corollary 4. Let $\mathscr{A}$ be a triangular algebra. Let us assume that
(i) $\mathscr{Z}(e \mathscr{A} e)=\mathscr{Z}(\mathscr{A}) e$
(ii) $\mathscr{Z}(f \mathscr{A} f)=\mathscr{Z}(\mathscr{A}) f$

Then, any generalized Jordan derivation $F: \mathscr{A} \longrightarrow \mathscr{A}$ is of the from $F(x)=\lambda x+J(x)$ for all $x \in \mathscr{A}$, where $\lambda \in Z(\mathscr{A})$ and $J: \mathscr{A} \longrightarrow \mathscr{A}$ is a derivation.

Corollary 5. Every Jordan derivation of a unital prime algebra $A$ with a nontrivial idempotent $e$ is a derivation.

Algebras of all bounded linear operators.

Let $X$ be a Banach space over $\mathbb{C}$ of dimension greater than 1 . By $\mathfrak{B}=B(X)$, we denote the algebra of all bounded linear operators on $X . \mathfrak{B}$ contains nontrivial idempotent $e$ and hence can be presented in the form $\mathfrak{B}=e \mathfrak{B} e+e \mathfrak{B} f+f \mathfrak{B} e+f \mathfrak{B} f$, where $f=1-e$. Since $\mathfrak{B}$ is a prime algebra, $\mathfrak{B}$ satisfies (⿻). Note that $e \mathfrak{B} e$ and $f \mathfrak{B} f$ are algebras of all bounded linear operators and all $\mathfrak{B}, e \mathfrak{B} e, f \mathfrak{B} f$ are central algebras over $\mathbb{C}$. Therefore, $e Z(\mathfrak{B}) e=Z(e \mathfrak{B} e)=$ $\mathbb{C} e$ and $f Z(\mathfrak{B}) f=Z(f \mathfrak{B} f)=\mathbb{C} f$. Hence $\mathfrak{B}$ meets assumptions of Theorem 1 and we have.

Corollary 6. Let $X$ be a Banach space over $\mathbb{C}$, $\operatorname{dim} X \geq 2$. Then every generalized Jordan $n$-derivation $\mathcal{S}$ of $\mathfrak{B}$ is of the form $\mathcal{S}(x)=\lambda x+J(x)$ for all $x \in \mathfrak{B}$, where $\lambda \in \mathbb{C}$ and $J: \mathfrak{B} \longrightarrow \mathfrak{B}$ is a Jordan $n$-derivation.

We know that $\mathfrak{B}$ is a prime algebra and all $\mathfrak{B}, e \mathfrak{B} e, f \mathfrak{B} f$ are central algebras over $\mathbb{C}$. Thus, the assumptions of the Wang'result ([2], Corollary 4.5) are fulfilled. Hence, any Jordan derivation $J: \mathfrak{B} \longrightarrow \mathfrak{B}$ is a derivation. 
Corollary 7. Let $X$ be a Banach space over $\mathbb{C}, \operatorname{dim} X \geq 2$. Then every generalized Jordan derivation $\mathcal{S}$ of $\mathfrak{B}$ is of the form $\mathcal{S}(x)=\lambda x+J(x)$ for all $x \in \mathfrak{B}$, where $\lambda \in \mathbb{C}$ and $J: \mathfrak{B} \longrightarrow \mathfrak{B}$ is a derivation.

\section{Data Availability}

No data were used to support this study

\section{Conflicts of Interest}

The authors declare that they have no conflicts of interest.

\section{Acknowledgments}

This work was supported by the Key Projects of Natural Science Research in Anhui Province (Grant No. 2008085QA01), Youth fund of Anhui Natural Science Foundation (Grant No. KJ2019A0107), National Natural Science Foundation of China (No. 11901030), and Natural Science Foundation of Beijing Municipality (No. 1204034).

\section{References}

[1] Z. K. Xiao and F. Wei, "Commuting mappings of generalized matrix algebras," Linear Algebra and Its Applications, vol. 433, pp. 2178-2197, 2010.

[2] D. Benkovič, "Jordan derivations of unital algebras with idempotents," Linear Algebra Application, vol. 437, no. 9, pp. 2271-2284, 2012.

[3] D. Benkovič, "Generalized lie derivations of unital algebras with idempotents," Operators and Matrices, vol. 12, no. 2, pp. 357-367, 2018.

[4] Y.-B. Li, L. van Wyk, and F. Wei, "Jordan derivations and antiderivations of generalized matrix algebras," Oper. Matrices, vol. 7, pp. 2-5, 2013.

[5] X. F. Qi, Z. Guo, and T. Zhang, "Characterizing Jordan nderivations of unital rings containing idempotents," Bulletin of the Iranian Mathematical Society, vol. 46, no. 6, pp. 1639-1658, 2020.

[6] D. Benkovič, "Generalized Lie n-derivations of triangular algebras," Communications in Algebra, vol. 47, pp. 5294-5302, 2019.

[7] J.-H. Zhang and W.-Y. Yu, "Jordan derivations of triangular algebras," Linear Algebra and its Application, vol. 419, no. 1, pp. 251-255, 2006.

[8] M. Brešar, "Jordan mappings of semiprime rings," Journal of Algebra, vol. 127, pp. 218-228, 1989.

[9] M. Brešar, "Jordan derivations revistited," Mathematical Proceedings of the Cambridge Philosophical Society, vol. 139, pp. 411-425, 2005.

[10] W.-S. Cheung, "Commuting maps of triangular algebras," Journal of the London Mathematical Society, vol. 63, no. 1, pp. 117-127, 2001.

[11] D. Benkovič, "Jordan derivations and antiderivations on triangular matrices," Linear Algebra and its Application, vol. 397, pp. 235-244, 2005.

[12] Y. Wang and Y. Wang, "Multiplicative lie $n$-derivations of generalized matrix algebras," Linear Algebra and Its Applications, vol. 438, no. 5, pp. 2599-2616, 2013.

[13] Y. Wang, "Lie $n$-derivations of unital algebras with idempotents," Linear Algebra Application, vol. 458, pp. 512-525, 2014.
[14] Y. N. Yan and J. Li, "Characterizations of Lie n-derivations of unital algebras with nontrivial idempotents," Filomat, vol. 32, pp. 4731-4754, 2018.

[15] W.-S. Cheung, Maps on triangular algebras, Ph.D. Dissertation, University of Victoria, Victoria, Canada, 2000.

[16] W.-S. Cheung, "Lie derivations of triangular algebras," Linear and Multilinear Algebra, vol. 51, no. 3, pp. 299-310, 2003.

[17] I. N. Herstein, "Jordan derivations of prime rings," Proceedings of the American Mathematical Society, vol. 8, no. 6, p. 1104, 1957.

[18] N. Jacobson and C. E. Rickart, "Jordan homomorphisms of rings," Transactions of the American Mathematical Society, vol. 69, p. 479, 1950. 